Voix et Images

volxetimages

\title{
Pour en finir avec un fantôme. Analyse manuscriptologique de Pour en finir avec Octobre de Francis Simard
}

\section{Claude Lebuis}

Volume 12, numéro 3 (36), printemps 1987

Yves Beauchemin

URI : https://id.erudit.org/iderudit/200660ar

DOI : https://doi.org/10.7202/200660ar

Aller au sommaire du numéro

Éditeur(s)

Université du Québec à Montréal

ISSN

0318-9201 (imprimé)

1705-933X (numérique)

Découvrir la revue

Citer cet article

Lebuis, C. (1987). Pour en finir avec un fantôme. Analyse manuscriptologique de Pour en finir avec Octobre de Francis Simard. Voix et Images, 12(3), 470-485. https://doi.org/10.7202/200660ar d'utilisation que vous pouvez consulter en ligne. 


\title{
Pour en finir avec un fantôme Analyse manuscriptologique de Pour en finir avec Octobre de Francis Simard 1
}

\author{
par Claude Lebuis, Université du Québec à Montréal
}

\begin{abstract}
Jai reçu les épreuves. Ils ont tout changé. Il y avait une lettre de la personne qui a fait les corrections /.../ Elle se vantait de m'avoir traduit du français au français. Vous voyez ce que je veux dire. Elle a passé le texte à l'eau de Javel, elle a rincé mon propos. C'est terrible. II ne reste plus rien.
\end{abstract}

Jacques SAVOIE ${ }^{2}$

1 Petite histoire d'un tapuscrit, de son scripteur et d'un censeur aux allures de fantôme

Peu d'études ont jusqu'ici décrit le rôle de réviseur/e-correcteur/e de manuscrit (ou "rewriter") dans les maisons d'édition québécoises. Pour Suzanne Robert et Jean-Pierre Leroux ${ }^{3}$, les réviseurs, ces taupes de l'édition, ont pour ingrate tâche la révision et la correction, mais surtout la réécriture, des manuscrits retenus par l'éditeur. Suzanne Robert distingue trois «avenues» ou types d'ouvrage sur lesquels porte le travail de révision-correctionréécriture:

Il peut s'agir de littérature générale (romans, nouvelles, poésie, théâtre, essais, revues, livres pour enfants, bandes dessinées), de littérature pédagogique (ouvrages scientifiques, revues savantes, dictionnaires, manuels scolaires) ou d'ouvrages pratiques (livres de recettes, de psychologie populaire, de bricolage $)^{4}$.

Ce travail s'effectue avec ou sans le concours des auteurs. L'anonymat est de mise, certaines réputations de "grands écrivains" pouvant en souffrir...

C'est un véritable dossier noir que Robert et Leroux ont préparé: les réviseures - car les femmes sont majoritaires - travaillent à la pige, sans statut professionnel et pour une rémunération dérisoire. Pourtant, leur labeur est souvent immense, car les éditeurs, qui ne sont pas toujours sélectifs, retiennent des manuscrits de mauvaise qualité.

Une recherche personnelle ${ }^{5}$ menée de 1977 à 1980 , sur la sélection et la révision des manuscrits littéraires, n'en trace pas un tableau plus reluisant: les éditeurs québécois déplorent le peu de révision pratiquée sur les manuscrits, à cause d'un manque de fonds. Ils aimeraient accorder plus de temps à la révision ${ }^{6}$. Il appert que cette révision ne couvre généralement que des registres superficiels de la composition, comme l'orthographe ${ }^{7}$. L'article re- 
vendicateur de Robert et Leroux est peut-être l'indice que certaines maisons d'édition québécoises accordent aujourd'hui plus d'importance à une révision approfondie des manuscrits, du fait d'une "stabilité" financière accrue. En effet, naguère "novices», plusieurs éditeurs québécois ont atteint une certaine "maturité", de par la constitution et la consolidation de leur fonds littéraire. Ce fonds leur permet, d'une part, d'assurer leur survie et, d'autre part, de renforcer leur "réputation", leur «image de marque», ce qui n'est pas du tout négligeable pour des entreprises livrées au «libre marché» et à la politique de subvention des différents gouvernements.

Si auteurs et éditeurs reconnaissent l'importance du travail de révision, la formation d'une association de réviseurs serait tout indiquée. Ce regroupement pourrait négocier un contrat-type avec les éditeurs (comme l'a déjà fait, pour les auteurs, l'Union des écrivains québécois), afin d'assurer stabilité et dignité aux membres de la "profession». Il y a cependant un obstacle de taille: l'isolement dans lequel se trouvent les réviseurs qui ne se re-connaissent pas, ne serait-ce qu'à l'intérieur d'une même maison d'édition. C'est une affaire à suivre ${ }^{8}$.

La révision que nous analyserons ici porte sur un texte de la catégorie "littérature générale», section "essai-témoignage autobiographique». Elle a été pratiquée sur un tapuscrit ${ }^{9}$ de Francis Simard, et consiste en une réécriture complète opérée par un "ghost», en l'absence de son scripteur ${ }^{10}$. Le "ghost" (appelé aussi "nègre") est un rédacteur anonyme (comme son nom l'indique. et comme l'est tout réviseur qui se respecte) chargé, par un éditeur, soit d'écrire un livre à partir d'une proposition de départ, soit de réécrire un manuscrit jugé de mauvaise qualité.

Au début de l'hiver 1981-1982, Francis Simard propose à l'éditeur Stanké un projet de livre sur les événements d'octobre 1970: Simard veut rédiger un témoignage, avec ses tripes, une sorte de discours, visant le plus grand nombre, dans lequel il utiliserait une langue accessible ", semblable à la langue familière, parlée par la majorité des gens. L'éditeur accepte et s'entend verbalement avec Simard pour que le manuscrit ne subisse aucune transformation majeure.

Le dossier Simard est alors confié à un collaborateur de Stanké, à qui Simard remet un tapuscrit d'une centaine de pages au printemps 1982. Comme Simard lui confie que le travail de rédaction est ardu, ce même collaborateur lui propose de laisser un "ghost» finir le travail: On va réécrire ton chapitre, écrire ton livre dans le style du premier chapitre. Dis-nous ce que tu veux mettre dedans. Simard refuse.

Quelques semaines plus tard, le scripteur vient prendre connaissance du rapport de lecture: il constate que le tapuscrit a été entièrement revu, réécrit. Le lecteur avait aussi fait office de réviseur. Simard ne reconnaît plus son style: d'une écriture proche de la parole familière, on est passé à un style plus "littéraire»; certaines adjonctions, selon Simard, déforment sa pensée. 
Simard avise Stanké. L'éditeur nommera alors un nouveau lecteurréviseur, qui sera chargé de lire le manuscrit et de proposer des corrections au scripteur: pas question de réécrire le texte. Le travail de parachèvement se fera désormais en équipe. Pour en finir avec Octobre ${ }^{12}$ paraîtra à l'automne 1982.

Les transformations apportées au manuscrit initial, toutes acceptées par Simard, auront été mineures: principalement des corrections orthographiques et, à plusieurs endroits, des substitutions du "on" par le "nous". L'éditeur aura respecté son engagement, ce que Simard tiendra à signaler en début d'ouvrage:
Je tiens à souligner $/ \ldots /$ le travail de l'éditeur qui, comme convenu, a respecté mon texte sans y apporter ni demander aucun changement ${ }^{13}$.

Seules les corrections du rédacteur-fantôme seront analysées dans les pages qui suivent. Le fait qu'elles n'aient pas été retenues pour la publication n'enlève rien à leur intérêt. L'analyse manuscriptologique peut y déceler nombre de présupposés stylistiques et idéologiques et jeter ainsi un peu de lumière sur cette instance méconnue de notre appareil d'édition.

Composé d'une centaine de pages, notre tapuscrit est divisé en deux sections foliotées respectivement de 1 à 58 et de 2 à 59 . La première section du tapuscrit correspond à la première partie (intitulée "Octobre") de la version publiée; la deuxième section tapuscrite correspond aux trois premiers chapitres de la deuxième partie éditée (intitulée "Avant"). Pour faciliter la description du tapuscrit, nous désignerons les deux sections à l'aide des lettres « $a$ » et « $b$ ». Par exemple, «39a» désignera la page 39 de la première partie tapuscrite.

Les corrections du rédacteur-fantôme se présentent de la façon suivante: on a rayé des passages à l'aide d'un feutre noir et réécrit à la mine au-dessus des rayures ${ }^{14}$.

Nous ne procéderons pas à l'étude d'un corpus restreint, mais plutôt à une analyse de l'ensemble du tapuscrit, les exemples cités servant à illustrer notre propos. Cette approche nous est apparue méthodologiquement nécessaire parce qu'elle permettait de dégager des constantes, des cohérences dans le travail de censure du correcteur-fantôme. Précisons que nous n'employons pas le mot «censure" dans son sens restreint d'acte d'interdiction, mais dans son acception latine plus large d'acte d'estimation, d'évaluation.

Ces constantes, nous les dénommerons «axes», terme qui traduit bien, il nous semble, l'idée que le réviseur imprime une certaine direction à sa pratique correctrice, en fonction de critères de correction auxquels il se renvoie constamment. Suzanne Robert indique, à juste titre, qu'une des qualités 
requises pour devenir "réviseur-correcteur-linguistique-de-manuscrits" est un sens du doute ${ }^{15}$ presque obsessionnel... Ajoutons que les axes révisionnels n'agissent pas séparément: ils s'appuient pour mieux faire effet.

Les opérations de base dans la révision d'un texte sont au nombre de quatre:

Tous les manuscriptologues s'accordent à distinguer les cas suivants: l'addition (ou ajout); la biffure sans remplacement du terme biffé (suppression) ou avec remplacement (substitution); le déplacement ou la permutation d'éléments. D'un point de vue linguistique, on peut considérer que toutes les variantes sont des substitutions (l'addition et la suppression ayant la particularité de comporter un terme nul) orientées par une chronologie et ne préservant pas le sens ${ }^{16}$.

Il va sans dire que ces quatre opérations jouent dans tous les axes ou paramètres de révision.

Tentative d'appropriation de la matière textuelle initiale du manuscrit par refaçonnement, l'opération de révision éditoriale pourrait être comparée au mécanisme d'élaboration secondaire que Freud identifie dans le rêve ${ }^{17}$. Composante de la censure, l'élaboration secondaire permet d'enlever à une production onirique son caractère d'apparente absurdité et d'incohérence, au moyen d'un choix opéré dans le matériel psychique des pensées du rêve. Par la restructuration qu'il impose au matériau textuel, le lecteur-réviseur s'efforce aussi, à sa façon, de donner à un texte cette cohérence qui lui semble faire défaut. L'élaboration secondaire du rêve est souvent coordination logique aveugle, naïve: par son effort excessif d'organisation, par son excès de zèle, le travail de censure se désigne, se trahit. Une situation semblable peut se produire lorsqu'un réviseur, "scripteur second", tente de modifier le cours initial de l'ouvrage: l'auteur ne s'y retrouve plus et il demande des comptes.

Toute révision éditoriale est le lieu d'une confrontation plus ou moins serrée entre un scripteur et son réviseur. Même si elle ne semble souvent opérer qu'au niveau d'une correction siylistiquée plưtôi innócente, la pratique révisionnelle s'appuie sur un ensemble complexe de valeurs, de présupposés, de goûts et de dégoûts ${ }^{18}$ dont le réviseur n'a pas nécessairement une conscience très nette et qui sont liés à sa position sociale: son origine sociale, sa "formation littéraire" et "culturelle», ses antécédents professionnels, sa position et sa "réputation» au sein de la maison d'édition, etc. Le réviseur ressemble bien à ce gardien dont nous parle Freud, qui voit à faire le tri dans le "matériau brut" (du rêve ou du texte), qui ne laisse pas passer n'importe quoi et qui, à tout moment, prend position ${ }^{19}$.

\section{Vue d'ensemble de l'entreprise de réécriture}

Simard considère que son .../ livre n'a aucune prétention littéraire. Avant d'être écrit, il a été vécu. Il veut rendre témoignage de ce vécu ${ }^{20}$. Pour ce faire, le scripteur pratique une écriture proche de l'oralité, simple, dépouil- 
lée, qui se caractérise principalement par son absence de variation lexicale. Des termes, que la stylistique traditionnelle et scolaire réprouve pour leur "banalité», abondent: le pronom "ça», les verbes "faire» et "avoir», etc.

Le lecteur-réviseur-fantôme, manifestement, ne prise pas du tout ce parti pris de simplicité: il ne peut admettre que le scripteur ne joue pas mieux son «jeu» d'auteur. Il décidera donc de mettre au pas littéraire le matériau textuel, de lui donner une allure plus conforme à ce qu'on est «en droit" d'attendre d'un texte qui réclame publication. "Professionnel de l'écriture", le "ghost" ira puiser à même l'arsenal de la bonne écriture et il opposera au scripteur toute une batterie d'armes éprouvées: des «beaux effets" de style.

Pour "revamper» l'écriture du scripteur, le réviseur-fantôme adoptera principalement deux stratégies ou axes révisionnels qui s'épauleront, se renforceront: un axe stylistique et un autre dramatique. Les corrections pratiquées sur l'axe stylistique visent essentiellement le lexique: en éliminant les termes "banals" ou en les substituant à des équivalents plus "littéraires", on tentera de «rehausser» le niveau de langue. Le travail de révision sur l'axe dramatique, de son côté, consiste à "enrichir» le texte d'informations à caractère romanesque, mélodramatique, pour rendre le récit plus «palpitant ". Les deux axes seront abordés de façon segmentée, car l'effet révisionnel est disséminé et multiforme: nous identifierons dans chacun des axes des éléments, divers en apparence, mais finalement concurrents.

Le corpus cité en exemple sera toujours présenté de la façon suivante: d'abord le texte original du tapuscrit de Simard (identifié par le mot "SIMARD»); ensuite, le texte réécrit par le "fantôme" ("FANT"); enfin, le texte publié par Stanké ( $S$ STANKÉ»). Les folios seront indiqués comme suit: "P. TAP», pour le tapuscrit de Simard et «P. STAN», pour l'édition Stanké. Sauf indication contraire, les soulignés sont toujours de nous.

\section{L'axe de correction stylistique}

On peut subdiviser l'axe de correction stylistique en quelques éléments principaux, question de mieux l'appréhender :

1- substitution des «mots banals" par un terme plus précis ou un synonyme;

2- correction d'ordre syntaxique et "déformation» du message initial;

3- addition de termes «joual» ou argotiques (français): une «contradiction stylistique".

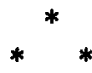

On peut dire que l'opération de réécriture fantôme consiste d'abord à hausser le niveau de la langue écrite, selon les distinctions traditionnelles de la stylistique scolaire: il s'agit de passer d'une langue "orale-ouvrière", considérée comme déficiente, à une langue "littéraire-policée» ("soutenue»). 
La langue "simple" que Simard a choisie ne semble pas "digne" de livrer un message sur une page importante de notre histoire nationale: les "événements" d'octobre 1970. Une transmutation, un "saut qualitatif» s'avère nécessaire et s'opérera prińcipalement par substitution de termes ou d'expressions considérés comme banáls, répétitifs et imprécis, par d'autres plus "colorés", assurant une certaine variété lexicale, fleuron glorieux du discours littéraire qui ne saurait souffrir de monotonie. Examinons tout d'abord quelques exemples de substitution du "ça", pronom démonstratif et «banal». Le scripteur ne tiendra aucun compte de ces corrections pour la version éditée.

SIMARD: traduite comme ça

FANT: traduite en ces termes

STANKE: id. Sim

P. TAP: 11 a P. STAN: 26

SIMARD: même l'enlèvement que voulait faire la cellule Libération, s'explique par ça.

FANT: / .../ s'explique dans cet esprit.

STANKÉ: id. Sim

P. TAP: $12 \mathrm{a}$ P. STAN: 27

Un autre type de révision du rédacteur-fantôme porte sur les mots banals (noms et verbes) remplacés par des termes plus précis. Encore là, même logique de "revampage» du niveau de langue:

SIMARD: Le monde les avaient tout volé les trottoirs.

FANT: Les citoyens avaient volé tous les trottoirs.

STANKÉ: Le monde avait tout volé les trottoirs.

P. TAP: $20 \mathrm{~b}$ P. STAN: 91

SIMARD: Tout ce que nous avions fait jusque là avait été fait en fonction de ça

FANT: Tout ce que nous avions accompli jusque là l'avait été en fonction de cet objectif.

STANKÉ: id. Sim / ortho: «jusque-ià» /

P. TAP: $12 \mathrm{a}$ P. STAN: 27

SIMARD: c'était tout ce qu'on avait

FANT: c'était tout ce que nous possédions

STANKÉ: c'était tout ce que nous avions

P. TAP: $42 \mathrm{a}$ P. STAN: 55

On notera, dans le dernier exemple, que Simard a permuté le pronom indéfini «on» au profit du pronom personnel «nous», pour la version éditée. Cette substitution s'est produite très fréquemment dans le texte. C'était une révision qui, aux yeux du scripteur, ne nuisait pas au style parlé du témoignage.

Le remplacement de certains termes redondants (le nom «Londres", dans l'exemple qui suit) fait aussi partie de la démarche correctrice: 
SIMARD: trois cent chambres bien situées au cœur de Londres.

FANT: trois cents chambres bien situées au cœur de la capitale anglaise. STANKÉ: trois cents chambres / etc. id. Sim /

P. TAP: $58 \mathrm{~b} \quad$ P. STAN: 127

Encore une fois, Simard rejette l'« offre» du rédacteur-fantôme: utiliser un synonyme. Notons que le syntagme de remplacement ("capitale anglaise») est éloigné de la parole familière. Un autre exemple illustre la recherche, par le "ghost", de termes précis:

SIMARD: un vingt dollars

FANT: un billet de vingt dollars

STANKE: un vingt dollars

P. TAP: $42 \mathrm{a} \quad$ P. STAN: 55

D'autres corrections, d'ordre syntaxique, illustrent bien la volonté du réviseur-fantôme de donner au texte un niveau plus "soutenu»:

SIMARD: j'avais décidé de piquer à travers les champs

FANT: j'avais décidé de piquer à travers champs

STANKE: j'avais piqué à travers les champs

P. TAP: $6 \mathrm{a}$ P. STAN: 21

SIMARD: C'est cette perspective-là qui nous semblait être la seule importante.

FANT: / ... qui nous semblait être la seule à avoir quelque importance.

STANKE: id. Sim

P. TAP: $12 \mathrm{a} \quad$ P. STAN: 27

Certaines corrections syntaxiques vont parfois «détourner», "déformer» le message du scripteur, bref provoquer des pertes d'information. Citons-en un seul exemple, qui semble au premier abord une correction anodine, mais qui renverse finalement la proposition initiale:

SIMARD: Ce n'est que ça qui me motive à parler ainsi. C'est ce que j'ai vécu. C'est ce que je crois qu'il a vécu.

FANT: Ce n'est pas seulement ça qui me motive à parler ainsi. / ... /

STANKE: Ce n'est que cela qui me motive à parler ainsi. $/ \ldots /$

P. TAP: $45 \mathrm{a}$ P. STAN: 58

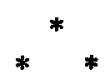

Une autre pratique révisionnelle, au niveau stylistique, consiste à ajouter des «faits de langue» québécois, question de faire couleur locale. Cette correction semble pourtant aller en contradiction avec le dessein premier de «rehausser» le niveau du texte. En voici quelques exemples:

SIMARD:C'était celle / une image/ d'un fonctionnaire, d'une affaire bureaucratique. 
FANT: C'était celle d'un fonctionnaire, d'une gamique bureaucratique. STANKÉ: id. Sim.

P.TAP: 22 b P. STAN: 93

SIMARD: Le parti c'est à eux.

FANT: Le Parti c'est à eux, c'est leur bébelle.

STANKE: id. Sim.

P.TAP: 43b P. STAN: 113

SIMARD: C'était démocratique à plein

FANT: C'était démocratique au coton

STANKE: id. Sim.

P.TAP: 43b P. STAN: 113

Étrangement, le rédacteur-fantôme semble donner raison au scripteur dans son parti pris d'oralité, étant donné que lui-même «en rajoute». Ailleurs, pourtant, il élimine d'autres expressions à saveur locale:

SIMARD: Il y a l'idée, la menterie qu'on nous conte.

FANT: Il y a l'idée, les mensonges qu'on nous conte

STANKË: id. Sim.

P.TAP: 36b P. STAN: 106

SIMARD: On était pas gros et pas mal perdus.

FANT: Nous n'en menions pas large et étions pas mal perdus.

STANKÉ: Nous n'étions pas gros et pas mal perdus.

P.TAP: 56 b P. STAN: 126

Cherchant tout à la fois à littéraliser et à oraliser l'écriture, la démarche révisionnelle tend à faire perdre son homogénéité à l'écriture volontairement simple du scripteur. Le choix de quelques termes relevant plus de l'argot "parigot» que du «joual» montréalais viendra renforcer cette tendance:

SIMARD: Bref, tout pour avoir le flux.

FANT: Bref, tout pour avoir le foira.

STANKÉ: id. Sim.

P. TAP: 22b P. STAN: 93-94

On constate donc à quel point le «discours» initial du scripteur est "perturbé» par l'intervention du rédacteur-fantôme.

\section{La dramatisation et son effet idéologique}

Le rédacteur-fantôme veut non seulement améliorer la phrase, le style, mais il veut aussi donner plus de "saveur" au sujet. Enlèvement, séquestration et mort d'une personnalité politique, enquêtes policières, intervention de l'armée: tous les ingrédients d'un récit "enlevant» sont réunis. On sait que les éditeurs sont friands de tels manuscrits: les témoignages autobiographiques, basés sur l'histoire "réelle et vécue" (par exemple, Papillon, d'Henri Charrière) connaissent souvent plus de succès que les romans, qui sont pourtant de gros "vendeurs» 21 . Tout en conservant une certaine facture romanesque 
(récit à intrigues, nombreux rebondissements de l'action), les récits autobiographiques ont l'avantage d'être "vrais". Le malheur avec Pour en finir avec Octobre, c'est que son scripteur, par son choix délibéré d'une écriture "simple", "nuit" à son propos, n'insiste pas assez sur le côté tragique de la situation.

Les corrections consisteront essentiellement à accentuer le côté dramatique (et accrocheur) des "événements» par substitution et adjonction d'informations: d'une part, on substituera des termes trop neutres par d'autres plus chargés émotivement, plus connotés; d'autre part, on ajoutera des commentaires venant renforcer une exposition trop faible.

La dramatisation de l'action comporte une dimension idéologique de taille : assombrir l'éclairage sur la "Crise d'Octobre $70 »$, c'est prendre position, c'est porter un jugement sur cet événement. En insistant sur les sentiments des protagonistes, en mettant le doigt sur la gravité de la situation, on arrive, par effet d'accumulation d'informations, à déplacer puis à inverser la portée idéologique initiale: on passe d'un récit qui se veut illustration et défense, en des termes «simples", d'une conviction politique (la nécessité de l'indépendance du Québec, pour et par les travailleurs), à un récit condamnant, parfois à mots couverts, parfois de façon explicite, les actes de terroristes présentés comme de violents doctrinaires. On comprend alors la réaction de Francis Simard. Jugeons-en par une vingtaine d'exemples, répartis en quatre rubriques: l'événement en lui-même (Octobre), les personnages secondaires, le révolutionnaire (son portrait) et la politique (sa mise en évidence). Nous rappelons que les soulignés sont toujours de nous.

\section{L'événement: octobre 1970}

Les corrections apportées ont pour but évident de donner plus de «relief» à l'“événement» (cet innommable), en identifiant de façon plus explicite les personnages, en mettant en relief leurs actions, en marquant le texte de moments forts (dans nos exemples, au moyen d'adverbes), le tout sur un ton souvent mélodramatique.

L'innommable: l'enlèvement et ses conséquences

SIMARD: À partir. de l'enlèvement de Pierre Laporte / ... /

FANT: A partir de cet acte /.../

STANKE: /id. Sim/

P. TAP: $34 \mathrm{a} \quad$ P. STAN: 47

Identification plus explicite des personnages et détails sur leur " vẹcu»"

SIMARD: On l'a pris dans nos bras.

FANT: On a pris le corps dans nos bras /... 
STANKÉ: Nous l'avons pris dans nos bras.

P. TAP: $56 \mathrm{a}$ P. STAN: 68

SIMARD: On exerçait toujours une surveillance.

FANT: Nous exercions toujours une surveillance sur le captif.

STANKÉ: Nous exercions toujours une surveillance.

P. TAP: $42 \mathrm{a}$ P. STAN: 55

SIMARD: Il est dans le salon, assis sur une chaise.

FANT: Il est dans le salon, assis sur une chaise, prostré.

STANKÉ: Id. Sim

P. TAP: $54 \mathrm{a}$ P. STAN: 66

SIMARD: le bandeau qui lui cachait la vue

FANT: le bandeau qui l'aveuglait

STANKÉ: Id. Sim

P. TAP: $45 \mathrm{a}$ P. STAN: 58

SIMARD: Tu sens que tu vas franchir de quoi...

FANT: Tu sens que tu vas franchir une porte fatidique.

STANKÉ: Tu sens que tu vas franchir quelque chose...

P. TAP: 25 a P. STAN: 39

SIMARD: ce sont ces impressions, ces sentiments, ces sensations qui m'étaient apparues...

FANT: ce sont ces impressions, ces sentiments, ces sensations qui m'envahissaient, me submergeaient.

STANKÉ: ce sont les impressions, les sentiments, la sensation qui m'étaient apparus...

P. TAP: 45 a P. STAN: 58

SIMARD: Si j'ai fait et passé au travers beaucoup de choses FANT: Si j'ai fait ce que j'ai fait et passé à travers beaucoup d'épreuves STANKE: Si j'ai fait et passé à travers beaucoup de choses

P. TAP: 6 a P. STAN: 21

SIMARD: J'pensais jamais que j'irais un jour. /en prison/

FANT: Je ne pensais jamais que l'on m'y enfermerait un jour.

STANKÉ: Je ne pensais jamais que j'irais un jour.

P. TAP: $51 \mathrm{~b}$ P. STAN: 121

\section{Marqueurs adverbiaux d'insistance}

Ici, les ajouts d'adverbes, en plus de convertir le texte à la norme stylistique de l'oralité, cherchent à donner un caractère tragique au récit, sur le ton de l'évidence pathétique:

SIMARD: Il a vraiment craqué.

FANT: Oui, il a vraiment craqué.

STANKÉ: id. Sim

P. TAP: $45 \mathrm{a} \quad$ P. STAN: 58 
SIMARD: On le libère ou on ne le libère pas.

FANT: Allons-nous le libérer, ouj ou non?

STANKÉ: Nous le libérons ou nous ne le libérons pas.

P. TAP: 52a P. STAN: 65

Les "personnages» secondaires

Le ton de la description est nettement mélodramatique:

SIMARD: Pour moi, l'exploitation, la misère, la violence, ça a un visage, celui de ces deux filles-là.

FANT: Pour moi, l'exploitation, la misère, la violence possèdent un visage: celui de ces deux malheureuses.

STANKE: id. Sim.

P. TAP: 50b P. STAN: 120

\section{Le portrait du révolutionnaire idéal}

Examinons maintenant des corrections très importantes, car elles contribuent, pour une large part, à inverser la portée idéologique du texte de Simard. C'est la connotation qui joue dans ces révisions principalement augmentatives. Les mots choisis ont une résonance très particulière, rappellent la "langue de bois" des textes de propagande soviétique: leitmotive de discours "révolutionnaires", au marxisme doctrinaire et à la logique implacable; signes de cruauté, apanage des "terroristes", en particulier des "felquistes"; discours de militant indépendantiste, évidemment intolérant et railleur. Bref, tous les traits que l'opinion commune prête aux forces politiques dites " de gauche». C'est un véritable tableau de chasse doxologique.

\section{Révolutionnaire et marxiste}

SIMARD: C'est toujours notre vie.

FANT: C'est toujours notre vie, notre lutte.

STANKÉ: id. Sim

P. TAP: 55 b P. STAN: 125

SIMARD: La réalité pour moi, ce n'était pas le brillant hall d'entrée, c'était elles.

FANT: La réalité pour moi, ce n'était pas le somptueux hall d'entrée, c'était ces sous-prolétaires.

STANKÉ: La réalité, pour moi, ce n'était pas le brillant du hall d'entrée, c'était elles.

P. TAP: 58 b P. STAN : 128

SIMARD: le F.L.Q. s'est présenté à nous comme le seul moyen d'être avec le monde.

FANT: / .../ d'être avec le vrai monde.

STANKÉ: le F.L.Q. s'est présenté à nous comme un moyen $/ \ldots /$

P. TAP: 48 b P. STAN: 118 
SIMARD: Il / un ami écossais/ connaissait Londres comme le fond de sa poche. Le vrai Londres, celui du monde.

FANT: / .../ Le vrai Londres, celui du monde ordinaire, du prolétariat. STANKÉ: / paragraphe éliminé/

P. TAP: 57b P. STAN: nil

SIMARD: Même si tu as les trois autres autour de toi, avec toi, tu es tout seul.

FANT: Même si tu as les trois autres camarades autour de toi, avec toi, tu es tout seul dans le fond.

STANKÉ: id. Sim.

P. TAP: 25a P. STAN: 39

\section{Le révolutionnaire doctrinaire}

Une simple substitution ou adjonction de conjonction peut produire un changement sémantique important. Dans les exemples suivants, le ton relève de la démonstration dogmatique:

SIMARD: Et le chantage s'exerce par ceux qui ont le pouvoir, $/ \ldots /$

FANT: Car le chantage s'exerce par ceux qui ont le Pouvoir $/ \ldots /$

STANKÉ: Et le chantage s'exerce par ceux qui ont le pouvoir.

P. TAP: $10 \mathrm{a}$ P. STAN: 25

SIMȦRD: On nous force à vivre dans un monde qui est comme ça.

FANT: Parce qu'on nous force $/ \ldots$ /

STANKÉ: / passage éliminé/

P. TAP: 31 a P. STAN: nil

\section{Le terroriste révolté et violent}

SIMARD: notre misère, notre pauvreté, on peut l'enlever à ceux qui la font, en profitent, pour s'en sortir... la faire disparaître.

FANT: il y avait moyen d'en sortir, de les faire disparaître en crachant à la face de ceux qui en sont responsables...

STANKE: id. Simard, sauf adjonction du pronom "qui» à "qui en profitent"

P. TAP: $13 \mathrm{a} \quad$ P. STAN: 28

SIMARD: / $\ldots /$ tu ne pars pas en pleine exaltation la baboune fendue jusqu'aux oreilles, ça n'a jamais été comme ça.

FANT: $/ \ldots /$ tu ne pars pas à l'attaque, en pleine exaltation, la «baboune» fendue jusqu'aux oreilles. Ça ne s'est jamais passé comme

STANKÉ: id. Sim

P. TAP: 25 a P. STAN: 39

SIMARD: Ce qui se présente le plus naturellement, parce-que / sic/ c'est un enlèvement qui a déclenché toute la crise, c'est d'en faire un autre. 
FANT: / ... parce que c'est un enlèvement qui a déclenché toute la Crise, c'est d'en perpétrer un autre.

STANKE: id. Sim, sauf trait d'union dans "parce-que»/

P. TAP: 10a P. STAN: 24-25

\section{L'indépendantiste vindicatif et railleur}

SIMARD: II / Pierre Laporte/ a oulié Pierre Elliot Trudeau et les membres du cabinet, la «toilette fédérale», qui eux décidèrent de refuser.

FANT: Il a oublié / .../ Pierre-Elliot Trudeau / .. / la «toilette fédéraste" $/ \ldots /$

STANKÉ: id. Sim

P. TAP: $43 a$ P. STAN: 56

\section{Mise en évidence du politique}

Étrangement, d'autres substitutions pourraient laisser entendre que les protagonistes felquistes ont un respect certain pour l'autorité en place:

SIMARD: On faisait attention. On l'a pris comme pour le sauver, $/ \ldots /$ FANT: Oui, nous faisions attention au ministre comme pour le sauver,... STANKÉ: Nous faisons attention. Nous l'avons pris comme pour le sauver, / .../

P. TAP: $56 \mathrm{a} \quad$ P. STAN: 68

De plus, le rédacteur-fantôme suggère au scripteur, par un double souligné à la mine, de placer des majuscules sur plusieurs mots, entre autres à Gouvernement, Système, Sciences sociales, Crise /économique/, Parti, Conseil des ministres, Pouvoir, Indépendance, etc. On peut se demander si, par ce procédé, le rédacteur-fantôme ne veut pas implicitement donner plus de poids à certaines réalités politiques (institutions et idées): d'une part, en prêtant au «narrateur Simard » une certaine reconnaissance de l'autorité politique; d'autre part, en nous présentant ce même "personnage" sous les traits d'un militant galvanisé, exalté par la "Cause» (politique) de l'Indépendance. Le scripteur ne tiendra pas compte de la plupart de ces «suggestions", sauf pour certains termes, comme: Loi des mesures de guerre, Octobre, Front de Libération, Révolution tranquille...

Le cas que nous venons d'analyser relève d'une sorte d'erreur d'aiguillage. Le problème réside dans le fait que le scripteur, Francis Simard, n'a pas été consulté en cours de production. Une telle révision, avec l'orientation qu'on lui connaît maintenant, n'aurait pu se produire si Simard avait d'emblée participé aux travaux de révision. On ne peut blâmer le réviseur, fantôme qui n'a fait que son travail. On ne peut non plus, à partir d'un simple cas d'espèce, 
généraliser et porter un jugement désapprobateur sur la révision de manuscrit. Notre intention n'était pas de montrer un «méchant réviseur» à l'œuvre, mais bien plutôt de démontrer à quel point la révision peut avoir une incidence profonde sur la «teneur» initiale d'un manuscrit. La révision éditoriale fäit partie de la physique de l'édition ${ }^{22}$ : elle est une étape cruciale, incontournable, du processus de publication. En cela, elle mérite d'être traitée avec une très grande attention.

1. Je tiens à remercier le scripteur, Francis Simard, pour sa précieuse collaboration, ainsi qu'un ami, Alain Bissonnette, avocat et anthropologue, qui m'a mis en contact avec le scripteur.

2. Jacques Savoie, "Marco Polo, ou le nouveau livre des merveilles", Épisode 1 : "L'angoisse amniotique», Le Devoir, 16 juillet 1985, p. 8.

3. Suzanne Robert et Jean-Pierre Leroux, "les Taupes de l'édition», Liberté, no 162, vol. 27, no 6 (décembre 1985), p. 3-16.

4. Suzanne Robert, loc. cit., p. 5 .

5. Claude Lebuis, la Censure éditoriale: sélection et révision des manuscrits littéraires, mémoire de maîtrise en Études littéraires, Université du Québec à Montréal, décembre $1980,379 \mathrm{p}$.

6. Ibid., p. 199.

7. Ibid., p. 332.

8. Ce paragraphe s'inspire des propos tenus par le réviseur Jean-Pierre Leroux, un des auteurs de l'article sur les "Taupes de l'édition", paru dans Liberté (conversation téléphonique du 28 novembre 1986).

9. Si le document est écrit à la main, c'est un manuscrit. S'il est dactylographié, c'est un dactylogramme, ou une dactylographie, ou, en formant un terme sur le modèle de manuscrit, un tapuscrit. [ Almuth Grésillon et Jean-Louis Lebrave, "Avant-propos" Langages, vol. 17, no 69 (mars 1983), p. 7, Thème du numéro: «Manuscrits-Eccriture, Production linguistique $"]$.

10. Nous préférons "scripteur" à "auteur»: Plus neutre qu'écrivain ou auteur, il ne préjuge pas de la qualité littéraire du document étudié et ne traîne après lui aucune connotation téléologique. (Grésillon et Lebrave, loc. cit., p. 8-9).

11. Propos de Francis Simard, en entrevue (printemps 1984).

12. Francis Simard, avec la collaboration de Bernard Lortie, Jacques Rose et Paul Rose; Pour en finir avec Octobre, Montréal, Éditions internationales Alain Stanké, 1982, $224 \dot{\mathbf{p}}$.

13. Simard, Pour en finir avec Octobre, p. 6.

14. Voir la reproduction de la page $13 \mathrm{a}$ du tapuscrit.

15. Suzanne Robert, loc. cit., p. 7.

16. Grésillon et Lebrave, loc. cit., p. 8.

17. Voir, entre autres ouvrages de Sigmund Freud, l'Interprétation des rêves, Paris, P. U.F., 1967, p. 417 sq.

18. /.../ les goûts sont sans doute avant tout des dégoûts, faits d'horreur ou d'intolérance viscćis!e ("c'est à vomir") pour les autres goûts, les goûts des autres. (Pierre Bourdieu, la Distinchion, Paris, Minuit, 1979, p. 60 (Coll. "Le sens commun»).

19. Pour plus d'informations sur les caractéristiques de la censure éditoriale, le lecteur peut consulter notre recherche, la Censure éditoriale, deuxième chapitre ("Quelques traits de la censure éditoriale $"$ ), plus pi. ticulièrement les « Repères théoriques", p. 1527 ainsi que «la Censure éditoriale: q1 . Iques repères», dans Moebius, numéro sur la censure, à paraître en 1987 . 
20. Simard et al., Pour en finir..., Avant-propos, p. 7.

21. Il est intéressant que cette catégorie "histoire et patrimoine" obtienne le plus gros tirage des ouvrages "littéraires". Ces ouvrages historiques peuvent même assurer la rentabilité de la maison d'édition, contrairement aux ouvrages purement fictionnels. /.../Ces /mêmes/ livres produisent une histoire à la portée de tous: leur facture littéraire de récit les rend plus faciles d'accès. L'histoire-patrimoine est une sorte de roman, en "plus vrai", parce qu' "historique». (Lebuis, la Censure éditoriale.... p. 71-72).

22. Nous faisons nôtre la définition brechtienne de la physique: /.../ une vision des mécanismes proprement dits, du pourquoi et du comment /.../ (Bertolt Brecht, «le Procès de quat'sous", "Expérience sociologique», Sur le cinéma, Paris, L'Arche, 1976, p. 172 (Coll. "Travaux", no 7). 


$$
\text { annaxe ouginat: }
$$

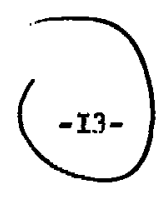

"réchapper" octobre. C'était la premièxe fois que le F.L.Q. entreprenait une action d'eclat, une action qui dépassait les frontières du Québec. Les médias d'information de par le monde en avatt par1e, brisant ainsi le glaçage du gâteau... (c'était la. glaçage de la démocratie canadienne. Première foigque le F.L.Q. fajsait connaftre un manifeste, fón texte qui cherbhait a traduire ce qu'était le F.L.Q., ses objectifs et qui êtait diffusé a l'ensemble de la population. Pour $=$ qu'octobre soit une prise de parole, ce n'étaj.t pas uniquement

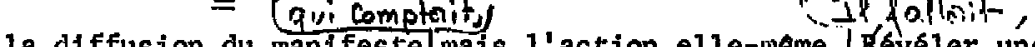
la diffusion du manifeste mais 1 action elle-meme.|Févéler une force: celle de ceux qui n'ont jamais la parole, Montrer une volonté d'en finir avec une societe de profiteurs, fusciter d'autres volontes de prendre la paro1e, de prendre en charge sa vie fen faisant un pes, fin montrant qu'i1 est possible d'en faire un. Montrer que rien n'est immable, établi. pour toujours. Wlotre sj.tuation d'exploite, notre misere, notre pauvrete, ily soudit mayen ol'os sortirj de les

Laire disparactre en crechant á la faca de ceux qui en Sont responsobles...

$$
* * *
$$

[Je ne sais plus qui ni quand, mais l'un Q'entré, est allé en vitesse acheter des perruques, de fausses moustaches, du maquillage, fó qu'il fallait pour se deguiser. Wous n'ovions patriotes. Seule la cellule Libération en avait. Elle 1'utillsait pour $=$ transmettre ses comminiques. On $s^{\dagger}$ est procure da ruban adhesif de couleur, 